\title{
Relationship between androgen levels and markers of endothelial dysfunction in men with type 2 diabetes
}

\author{
Irina Khripun, Igor Belousov, Sergey Vorobiev, Elizaveta Dzantieva, Zalina Gusova, Valentina Puzireva, \\ Asiat Sultanmuradova, Maria Haritonova \\ Rostov State Medical University, Rostov-on-Don, Russia
}

Background:

Objectives:

Methods:

Results:
It is well known that type 2 diabetes causes the endothelial dysfunction. However, insufficient attention is paid to the role of androgen deficiency in the formation of the early signs of endothelial dysfunction.

The aim of the work was to evaluate the relationship between testosterone deficiency and markers of endothelial dysfunction in men with type 2 diabetes.

Were examined 88 men aged 40-65 years (mean age 53,0 \pm 7,4 years) with type 2 diabetes. Clinical examination included the measurement of body weight, height, hip (HC) and waist (WC) circumferences and arterial blood pressure. A sample of fasting venous blood was taken to measure glucose, HbA1c, lipid profile, total testosterone, concentrations of ICAM-1, VCAM-1, resistin, e-selectin, p-selectin, C-reactive protein (CRP).

All patients were divided into two groups matched for age and levels of arterial blood pressure (table 1). The first group included 47 patients with normotestosteronemy, the second - 41 men with testosterone concentration below $12,1 \mathrm{nM} / \mathrm{L}$.

Statistical analysis was performed using analysis of the median Me [LQ; UQ] and comparison of two independent groups (Mann - Whitney $U$ test).

\section{Table 1. Clinical characteristics of the Picture 1.The concentration of} groups according to the level of $\quad$ resistin and CRP in two groups testosterone

\begin{tabular}{|c|c|c|c|}
\hline Parameter & Group 1 & Group 2 & P \\
\hline IMC, $\mathrm{kg} / \mathrm{m}^{2}$ & $30,0[22,7 ; 33,1]$ & $31,5[29,5 ; 36,9]^{*}$ & 0,023 \\
\hline $\mathrm{WC}, \mathrm{sm}$ & $107,0[101,0 ; 115,0]$ & $111,5[102,0 ; 127,0]^{*}$ & 0,049 \\
\hline $\mathrm{HC}, \mathrm{sm}$ & $108,0[102,5 ; 114,0]$ & $115,0[108,0 ; 120,0]^{*}$ & 0,047 \\
\hline SBP, mm Hg & $139,0[130,0 ; 151,0]$ & $141,0[136,0 ; 150,0]$ & 0,329 \\
\hline DBP, mm Hg & $84,0[80,0 ; 91,0]$ & $83,0[79,0 ; 92,0]$ & 0,692 \\
\hline TC, mM/l & $5,4[4,9 ; 6,9]$ & $5,7[4,7 ; 6,3]$ & 0,088 \\
\hline TG, mM/l & $1,7[1,2 ; 2,3]$ & $2,0[1,6 ; 3,0]^{*}$ & 0,031 \\
\hline HDL, mM/l & $1,4[1,3 ; 1,6]$ & $1,5[1,4 ; 1,6]$ & 0,091 \\
\hline VLDL, mM/l & $0,7[0,6 ; 1,0]$ & $0,9[0,7 ; 1,4]^{*}$ & 0,034 \\
\hline HbA1c & $7,5[6,3 ; 9,3]$ & $8,4[7,0 ; 11,7]^{*}$ & 0,049 \\
\hline
\end{tabular}
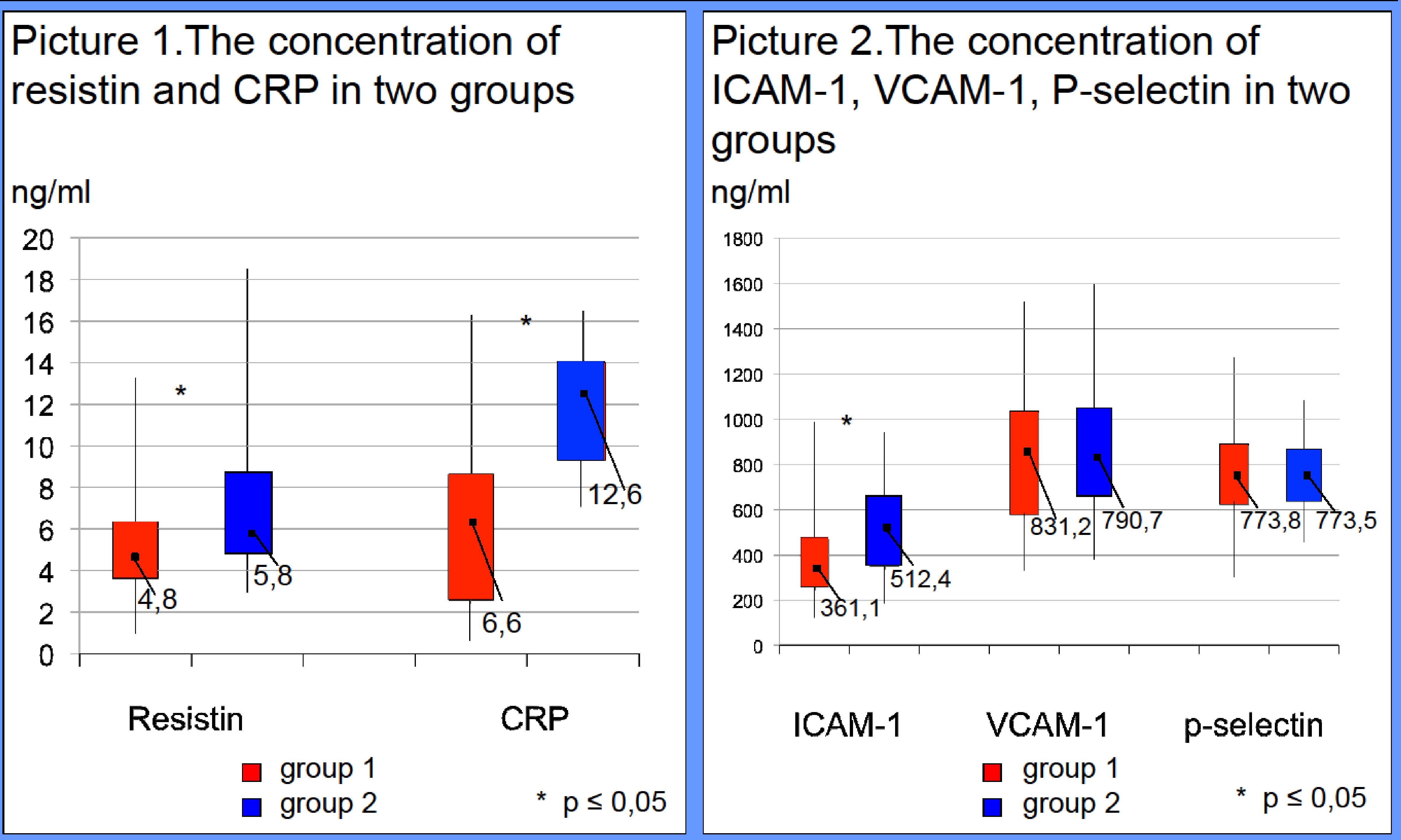

The analysis of the data shows that hypogonadal patients had a significantly higher body mass index (BMI), WC, HC. Also the levels of HbA1c, triglycerides (TG), very low dencity lipoproteins (VLDL) were statistically higher in patients of the second group. It indicates the deterioration of control of carbohydrate and lipid metabolism in the state of androgen deficiency.

We have not found statistically significant differences between the two groups in a concentration of E-selectin, P-selectin and VCAM-1. This may reflect a slight influence of hypotestosteronemy on these markers of endothelial dysfunction.

In the second group was found a significant increase of resistin $(\mathrm{p}=0,01): 5.81[5.05 ; 8.7] \mathrm{ng} / \mathrm{ml}$ vs 4,82 $[3,63 ; 6.34] \mathrm{ng} / \mathrm{ml}$ and ICAM-1 $(\mathrm{p}=0,04): 512.45$ [350.1; 644.4] ng / ml vs 361,15 [256,8; 478.5] ng / ml. In addition, was observed an increase in CRP levels in patients with late onset hypogonadism compared to eugonadal men $(12.65[10.9,14.00]$ vs $6,62[2,59 ; 8,61] \mathrm{mg} / \mathrm{L})(\mathrm{p}=0.0001)$.

Thus, in patients with a deficiency of testosterone were significantly higher levels of markers of endothelial dysfunction: resistin at $17 \%$ and ICAM-1 at $29.5 \%$; also the concentration of CRP at $47.7 \%$ compared to eugonadal men with type 2 diabetes.

The identified patterns suggest that in diabetes with androgen defficiency occur biochemical processes, reflecting the presence of endothelial dysfunction. Along with a synchronous increase in CRP level, this indirectly indicates a rise in cardio-vascular risk in this group of patients.

Established a significant increase in the concentrations of resistin and ICAM-1 in patients with type 2 diabetes and late onset hypogonadism may be the basis for the study of their role as predictors of the development of cardiovascular diseases at an early stage.

This work was supported by the Russian Science Foundation, grant № 14-25-00052. 